\title{
Recent results and perspectives on cosmic backgrounds from radio to far-infrared
}

Burigana, Carlo; De Zotti, Gianfranco; Fialkov, Anastasia; Qadir, Asghar; Trombetti, Tiziana; Bonato, Matteo; Negrello, Mattia; Nørgaard-Nielsen, Hans Ulrik; Tahir, Noraiz

Published in:

International Journal of Modern Physics D

Link to article, DOI:

$10.1142 / S 0218271819300210$

Publication date:

2019

Document Version

Peer reviewed version

Link back to DTU Orbit

Citation (APA):

Burigana, C., De Zotti, G., Fialkov, A., Qadir, A., Trombetti, T., Bonato, M., Negrello, M., Nørgaard-Nielsen, H. U., \& Tahir, N. (2019). Recent results and perspectives on cosmic backgrounds from radio to far-infrared. International Journal of Modern Physics D, 28(12), [1930021 ]. https://doi.org/10.1142/S0218271819300210

\section{General rights}

Copyright and moral rights for the publications made accessible in the public portal are retained by the authors and/or other copyright owners and it is a condition of accessing publications that users recognise and abide by the legal requirements associated with these rights.

- Users may download and print one copy of any publication from the public portal for the purpose of private study or research.

- You may not further distribute the material or use it for any profit-making activity or commercial gain

- You may freely distribute the URL identifying the publication in the public portal 


\title{
Recent results and perspectives on cosmic backgrounds from radio to far-infrared ${ }^{*}$
}

\author{
Burigana, Carlo; ${ }^{1,2,3, a}$ De Zotti, Gianfranco; ${ }^{4, b}$ Fialkov, Anastasia $;^{5,6, c}$ \\ Qadir, Asghar; ${ }^{7, d}$ Trombetti, Tiziana; ${ }^{1, e}$ Bonato, Matteo; ${ }^{1, f}$ \\ Negrello, Mattia $;^{8, g}$ Norgaard-Nielsen, Hans Ulrik $;^{9, h}$ Tahir, Noraiz ${ }^{7, i}$ \\ ${ }^{1}$ INAF-IRA, Via Piero Gobetti 101, I-40129 Bologna, Italy ${ }^{\dagger}$ \\ ${ }^{2}$ Dipartimento di Fisica e Scienze della Terra, Università degli Studi di Ferrara, \\ Via Giuseppe Saragat 1, I-44122 Ferrara, Italy \\ ${ }^{3}$ INFN, Sezione di Bologna, Via Irnerio 46, I-40126, Bologna, Italy \\ ${ }^{4}$ INAF-Osservatorio Astronomico di Padova, Vicolo dell'Osservatorio 5, I-35122 Padova, Italy \\ ${ }^{5}$ Harvard-Smithsonian Center for Astrophysics, 60 Garden Street, Cambridge, MA 02138, USA \\ ${ }^{6}$ Astronomy Centre, Department of Physics and Astronomy, University of Sussex, Brighton \\ BN1 9QH, UK \\ ${ }^{7}$ School of Natural Sciences, National University of Science and Technology, H-12 Islamabad, \\ 46000, Pakistan \\ ${ }^{8}$ School of Physics and Astronomy, Cardiff University, The Parade, Cardiff CF24 3AA, UK \\ ${ }^{9}$ DTU Space, Elektrovej, DK - 2800 Kgs. Lyngby, Denmark \\ aburigana@ira.inaf.it - ${ }^{b}$ gianfranco.dezotti@inaf.it - ${ }^{c}$ anastasia.fialkov@gmail.com \\ ${ }^{d}$ asgharqadir46@gmail.com - ${ }^{2}$ trombetti@ira.inaf.it - fbonateo@yahoo.it \\ ${ }^{g}$ NegrelloM@cardiff.ac.uk $-{ }^{h}$ hunn@space.dtu.dk $-{ }^{i}$ noraiztahir78637@gmail.com
}

Cosmological and astrophysical surveys from radio to far-infrared, in both temperature and polarization, offer a unique view of the Universe properties and of the formation and evolution of its structures. The last release, close to be finalized, of the Planck mission results sets the scene for cosmological models and parameters, while the comparison with other types of data sets raises the issue of possible tensions about some parameters, first of all the Hubble constant. At the same time, on the extragalactic side, Planck carried out the deepest systematic all-sky survey of SZ galaxy clusters and detected thousands of dusty galaxies and many hundreds of extragalactic radio sources, also allowing us to investigate many specific topics, including molecular hydrogen clouds in galactic halos. The exploitation of future generation of CMB missions and the next radio facilities will allow us to deeply investigate several topics in cosmology and astrophysics, from the existence of primordial gravitational waves to the energy releases in the primeval plasma, from the dawn ages and the epoch of reionization to the formation and evolution of early galaxies and clusters, while a wide set of open astrophysical problems can be studied with future IR missions.

Keywords: Cosmology; Background radiations; Radio, microwave; Origin and formation of the Universe; Observational cosmology; Large scale structure of the Universe; Galaxies.

\section{Introduction}

The past and recent surveys from radio to far-infrared have offered fundamental probes to answer to many questions of cosmology and on the formation and evolution of the cosmic structures at different scales and times. In particular, the accurate observations of cosmic microwave background (CMB) properties sanctioned

\footnotetext{
*Based on talks presented at the Fourteenth Marcel Grossmann Meeting on General Relativity, Rome, July 2015.

${ }^{\dagger}$ Istituto Nazionale di Astrofisica - Istituto di Radioastronomia, Via Piero Gobetti 101, I-40129 Bologna, Italy
} 
the passage to the so-called precision cosmology, calling for a further progress to unravel the mysteries of the early Universe and of its evolution up to the present epoch and beyond. At the same time, the information contained in the radio sky is of increasing relevance to unveil the complexity of the early stages of stars and galaxies stored in the imprints left in the cosmic background by the $21-\mathrm{cm}$ redshifted line from neutral hydrogen from the dark and dawn ages to the epoch of reionization (EoR), accessible thanks to the enormous improvement of recent and future radio projects and facilities. Sub-millimetre to infrared (IR) observations offer powerful diagnostics for a broad set of astrophysical mechanisms and conditions, also because, due to the reprocessing and the cosmic expansion, a large fraction of the optical and ultraviolet radiation from stars in galaxies comes to us at longer wavelengths. The exploitation of these surveys also revealed the close connection between our comprehension of the global properties of the Universe and the detailed understanding of the astrophysical processes operating in bounded objects and in the diffuse medium at different scales.

The structure of this paper is as follows. In Sect. 2.1 we report on some of the main cosmological results from the Planck mission, focussing on the precise determination of cosmological parameters in the $\Lambda$ cold dark matter $(\Lambda \mathrm{CDM})$ model and in some extensions, and discuss the issue of possible tensions about some parameters, first of all the Hubble constant. The estimation of a particular parameter combination based on the analysis of the diffuse thermal Sunyaev-Zeldovich (SZ) effect coming from all the galaxies clusters and the related so-called bias problem are discussed. In Sect. 3 we briefly describe recent proposals of future CMB missions and their main scientific purposes. Sect. 4 is dedicated to some specific topics in astrophysical cosmology, namely the study of CMB spectral distortions and of cosmic IR background (CIB) spectrum through the analysis of the cosmic dipole frequency behaviour aimed at constraining the (early and late) thermal and star formation histories, the cosmological evolution of neutral hydrogen at high redshifts through the analysis of the 21-cm redshifted line and the imprints of black holes high mass X-ray binaries microquasars in cosmic backgrounds. In Sect. 5 we move to extragalactic astrophysics. After a brief description of the Planck contribution in the field, we illustrate two new promising research paths, the study of early phases of galaxy evolution exploiting the strong gravitational lensing and of the cluster evolution, in relation to the prospects of future CMB experiments. We then report on an investigation of the molecular hydrogen clouds in galactic halos using Planck observations of the Doppler asymmetry in CMB radiation. We finally illustrate a wide set of open astrophysical problems that can be investigated with a future IR space mission. 


\section{Cosmological parameters}

\subsection{Planck results}

The Planck satellite ${ }^{\mathrm{a}}$, launched in 2009, was equipped with a Gregorian dual reflector telescope of $1.5 \mathrm{~m}$ diameter, whose focal plane was able to collect the microwave photons owing to two wide band cooled receiver arrays, the Low Frequency Instrument (LFI) made up of radiometers operative at $20 \mathrm{~K}$, and the High Frequency Instrument (HFI) composed of bolometers working at $100 \mathrm{mK}$. The two instruments observed the sky in 9 frequency bands, performing respectively 8 and 5 nearly all sky surveys, LFI at 30, 44 and $70 \mathrm{GHz}$ and HFI at 100, 143, 217, 353, 545, $857 \mathrm{GHz}$, with telescope optical axis pointing at $85^{\circ}$ from the spin axis ${ }^{1,2}$. Planck observed the sky from a large Lissajous orbit around the Sun-Earth L2 Lagrange point in order to offer stable observing conditions and minimize contamination from side lobe pick-up of stray radiation from the Sun, Earth, and Moon, scanning the sky while spinning at $1 \mathrm{rpm}$ in almost great circles. The Planck full width half maximum (FWHM) resolution ranges from $33.3^{\prime}$ to $4.3^{\prime}$ from $30 \mathrm{GHz}$ to $857 \mathrm{GHz}$, and the final sensitivity per $\mathrm{FWHM}^{2}$ resolution element span $\sim 2-14 \mu \mathrm{K} / \mathrm{K}$ in terms of $\delta T / T$ for frequencies $\nu \leq 353 \mathrm{GHz}$.

Reprocessing LFI and HFI data, the Planck Collaboration released maps in temperature at the nine frequency bands and in polarization at the seven polarization sensitive bands up to $353 \mathrm{GHz}$, and extracted Stokes $T, Q, U$ parameter maps of CMB anisotropies on the whole sky with unprecedented sensitivity and resolution. For many investigations, the anisotropy signals of billions of pixels are compressed in several thousand of numbers, namely the correlators $C_{\ell}^{X Y}$, where $X$ and $Y$ stands for $T, E, B$, at the multipole $\ell \sim 180^{\circ} / \theta, \theta$ being the angular scale of interest. A linear combination of $Q, U$ allows us to define, in Fourier space, the pure polarization angular power spectra (APS), the so-called $E$-modes (gradient component) and $B$ modes (curl component). Together with temperature anisotropies, four APS, $C_{\ell}^{T T}$, $C_{\ell}^{E E}, C_{\ell}^{B B}, C_{\ell}^{T E}$, the latter being the temperature polarization cross correlation, were extracted. Under the assumption of random Gaussian fluctuations, the CMB APS $C_{\ell}^{X Y}$ contain all the relevant statistical information that was indeed mainly analyzed through Markov chain Monte Carlo methods ingesting the corresponding theoretical predictions of Boltzmann codes to derive the cosmological parameters characterizing sets of models, marginalizing also over instrument and foreground residual parametrizations ${ }^{3}$.

Cosmological results based on Planck observations were presented in three (2013, 2015 and 2018) subsequent releases. The temperature and polarization CMB APS are consistent with the standard spatially-flat six-parameter $\Lambda$ CDM cosmology with

\footnotetext{
a Planck hosted instruments provided by two scientific Consortia funded by European Space Agency (ESA) member states (in particular the lead countries: France and Italy) with contributions from National Aeronautics and Space Administration (NASA), USA, and telescope reflectors provided in a collaboration between ESA and a scientific Consortium led and funded by Denmark.
} 
Table 1. Estimation and $68 \%$ C.L. intervals for the six-parameters of the base $\Lambda \mathrm{CDM}$ model and five derived parameters from the 2018 Planck release of CMB APS in $T T$ and low- $\ell E E$ modes (second column) and in $T T, E E$ and $T E$ modes in combination with $\mathrm{CMB}$ lensing reconstruction and $\mathrm{BAO}$ (third column).

\begin{tabular}{ccc}
\hline Six-parameters of the base $\Lambda$ CDM model & & \\
\hline$\Omega_{\mathrm{b}} h^{2}$ & $0.02212 \pm 0.00022$ & $0.02242 \pm 0.00014$ \\
$\Omega_{\mathrm{c}} h^{2}$ & $0.1206 \pm 0.0021$ & $0.11933 \pm 0.00091$ \\
$100 \theta_{\mathrm{MC}}$ & $1.04077 \pm 0.00047$ & $1.04101 \pm 0.00029$ \\
$\tau$ & $0.0522 \pm 0.0080$ & $0.0561 \pm 0.0071$ \\
$\ln \left(10^{10} A_{\mathrm{s}}\right)$ & $3.040 \pm 0.016$ & $3.047 \pm 0.014$ \\
$n_{\mathrm{s}}$ & $0.9626 \pm 0.0057$ & $0.9665 \pm 0.0038$ \\
\hline Derived parameters & & \\
\hline$H_{0}[\mathrm{~km} / \mathrm{s} / \mathrm{Mpc}]$ & $66.88 \pm 0.92$ & $67.66 \pm 0.42$ \\
$\Omega_{\mathrm{m}}$ & $0.321 \pm 0.013$ & $0.3111 \pm 0.0056$ \\
$\sigma_{8}$ & $0.8118 \pm 0.0089$ & $0.8102 \pm 0.0060$ \\
$10^{9} A_{\mathrm{s}} e^{-2 \tau}$ & $1.884 \pm 0.014$ & $1.881 \pm 0.010$ \\
Age of the Universe $[\mathrm{Gyr}]$ & $13.830 \pm 0.037$ & $13.787 \pm 0.020$ \\
\hline
\end{tabular}

a power-law spectrum of adiabatic scalar perturbations (denoted "base $\Lambda$ CDM").

Cosmological parameter results ${ }^{4}$ from the final full-mission Planck measurements ${ }^{5-7}$ have been presented in the 2018 release, combining information from the temperature and polarization maps and the lensing reconstruction and including the information from baryonic acoustic oscillations (BAO) (see Tab. 1). ${ }^{\mathrm{b}}$

Compared to the 2015 results $^{3}$, the improved measurement of large-scale polarization, foreground cleaning and likelihood analysis allow us to measure the value of $\tau$ (see also Sects. 4.1 and 4.2) with higher precision, suggesting a mid-point reionization redshift in the range $\simeq 7.1-7.8$ only slightly dependent on the explored data set (namely, $z_{\mathrm{re}}=7.50 \pm 0.82$ or $z_{\mathrm{re}}=7.8 \pm 0.7$ with reference to the cases in Tab. 1), in agreement with astrophysical observations of quasar absorption lines and supporting models in which reionization happened relatively fast and late. Extensions to more general reionization models indicate that the results found for the other cosmological parameters are essentially insensitive to uncertainties in the reionization history.

The refined analysis of the small-scale polarization provided a more robust determination of many parameters, the uncertainties in the treatment of various types of residuals affecting their estimation only at the $0.5 \sigma$ level. A remarkable, good consistency of the six parameters of the base $\Lambda \mathrm{CDM}$ model was found when considering

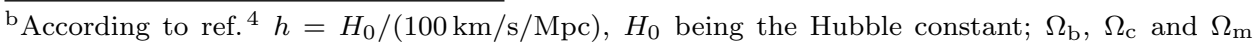
are respectively the baryon, cold dark matter (CDM) and (global, non-relativistic) matter density parameters; $\tau$ is the Thomson optical depth of cosmological reionization; $\theta_{\mathrm{MC}}$ is an approximation to the acoustic scale angle $\theta_{*} \equiv r_{*} / D_{\mathrm{M}}$ where $r_{*}$ is the comoving sound horizon at recombination quantifying the distance the photon-baryon perturbations can influence, and $D_{\mathrm{M}}=(1+z) D_{\mathrm{A}}$, where $D_{\mathrm{A}}$ is the usual angular diameter distance, is the comoving angular diameter distance that maps this distance into an angle on the sky; $A_{\mathrm{s}}$ and $n_{\mathrm{s}}$ are the amplitude and spectral index of the power spectrum of primordial scalar perturbations; $\sigma_{8}$ is the matter fluctuation amplitude on a scale of $8 h^{-1} \mathrm{Mpc}$.
} 
polarization, temperature, and lensing data, separately and in combination. Even considering many typical extensions of this model, the results found for these parameters remain substantially stable, with only a weak dependence on the adopted cosmological model and only slightly increased uncertainties in their estimation.

\subsection{Model extensions and the tension on the Hubble constant}

Various types of extensions of the base $\Lambda$ CDM model were considered in the Planck 2018 release, finding no compelling evidence supporting extensions to the base $\Lambda \mathrm{CDM}$ model.

Relativistic particle influence CMB anisotropies, mainly at intermediate and small angular scales. Planck data in combination with BAO measurements (and considering single-parameter extensions) constrain the effective extra relativistic degrees of freedom to be $N_{\text {eff }}=2.99 \pm 0.17$, in agreement with the Standard Model prediction $N_{\text {eff }}=3.046$, and tightly constrain the neutrino mass to $\sum m_{\nu}<0.12 \mathrm{eV}$.

Standard big-bang nucleosynthesis predictions for the helium and deuterium abundances for the base $\Lambda \mathrm{CDM}$ cosmology are in excellent agreement with observations.

The CMB spectra prefer higher lensing amplitudes than predicted in base $\Lambda$ CDM model at over $2 \sigma$, which pulls some parameters that affect the lensing amplitude away from the $\Lambda \mathrm{CDM}$ model; however, this is not supported by the lensing reconstruction $^{8}$ or (in models that also change the background geometry) BAO data.

The joint constraint with BAO measurements on spatial curvature is consistent with a flat universe, $\Omega_{K}=0.001 \pm 0.002$, while combining with Type Ia supernovae $(\mathrm{SNe})$, the dark energy (DE) equation of state parameter is measured to be $w_{0}=$ $-1.03 \pm 0.03$, with no significant deviations from an interpretation in terms of a cosmological constant.

At the scales measured by Planck the primordial spectrum has been found to be well described by a pure power-law. The joint analysis of CMB polarization from Planck, BICEP2 and Keck Array data, in combination with measurements from $\mathrm{BAO}$, sets the ratio between primordial tensor and scalar perturbations to $r<0.07$ at the wavenumber $k=0.002$, constraining inflationary models ${ }^{9}$ and setting the scene for future projects (see Sect. 3).

The Planck base $\Lambda$ CDM results are in good agreement with $\mathrm{BAO}, \mathrm{SNe}$, and some galaxy lensing observations, but in slight tension with the Dark Energy Survey's combined-probe results including galaxy clustering (which prefer lower fluctuation amplitudes or matter density parameters).

The value of the $H_{0}$ for the Planck base $\Lambda$ CDM cosmology results in substantial tension, at $\simeq 3.6 \sigma$ level, with the local determination through Type Ia SNe by ref. ${ }^{10}$ and even more, at $\simeq 4.4 \sigma$ level, with the most recent one by ref. ${ }^{11}$ which implies a higher value, $H_{0}=(74.03 \pm 1.5) \mathrm{km} / \mathrm{s} / \mathrm{Mpc}$, while the Planck measurement is in excellent agreement with independent inverse-distance ladder measurements using BAO, SNe, and element abundance results. Model extensions have been in- 
vestigated in ref. ${ }^{4}$ to resolve the tension with the value of $H_{0}$ from Type Ia SNe, including, other than those mentioned above, also modified gravity, DE models, recombination history, primordial scalar perturbations with running spectral index possibly combined with tensor modes, but none of these models convincingly resolves this problem.

An attempt to solve this tension involves the observational evidence of the existence of local radial inhomogeneities extending in different directions up to a redshift $z \simeq 0.07$ that could affect a significant fraction $(\sim 40 \%)$ of the Cepheids used for SNe calibration. Different methods used in ref. ${ }^{12}$ to compute the effects of these inhomogeneities on the low-redshift luminosity and angular diameter distance, using an exact solution of the Einstein's equations, linear perturbation theory and a low-redshift expansion, point towards the relevance of the non-relativistic Doppler redshift correction (proportional to the volume averaged density contrast and to the comoving distance from the center). Using a new direct relation between the luminosity distance and the monopole of the density contrast, which does not involve any metric perturbation, the monopole of the density field was reconstructed through a new inversion method from the deviations of the redshift uncorrected observed luminosity distance respect to the $\Lambda$ CDM prediction based on cosmological parameters obtained from large scale observations. The method suggests the existence of inhomogeneities effects not previously taken into account in density field maps at $z \leq 0.06$, used to obtain the peculiar velocity for redshift correction, i.e. at a scale not large enough for detecting inhomogeneities up to $z=0.07$, underlying the relevance of accurately normalizing the density field respect to the average large scale density of the Universe. Of course, the CMB high- $z$ measure of $H_{0}$ are insensitive to local inhomogeneities.

Other methods explore observations at intermediate redshifts. For example, in ref. ${ }^{13}$ the joint use of four different observables (SZ effect combined with X-ray surface brightness of galaxy clusters, ages of old high-redshift galaxies, observational measurements of the Hubble parameter, and BAO peak) proposed in ref. ${ }^{14}$ was revised reconsidering the sample of galaxy clusters and performing a new characterization of systematic uncertainties, and a value of $H_{0}$ fully consistent with Planck results was inferred.

Forthcoming cosmological surveys in various bands, including those aimed at filling the gap between low and high redshifts measures of $H_{0}$, will allow us to firmly understand if the tension comes from observational or data analysis issues or reflects instead the imprint of physics beyond $\Lambda \mathrm{CDM}$ possibly involving, e.g., specific neutrino sector ${ }^{15}$ or early $\mathrm{DE}^{16}$ models.

\subsection{Constraints from thermal $S Z$ power spectrum}

The Planck mission performed multifrequency surveys of the SZ effect, the Second Planck catalogue of SZ galaxy clusters (PSZ2) being the largest and deepest allsky selected galaxy clusters archive, enclosing 1653 sources $^{17}, \sim 73 \%$ of them with 
counterparts in external data sets (see also ref. ${ }^{5}$ ). Remarkably, the Planck surveys allowed us to study the diffuse thermal SZ (tSZ) effect coming from all the galaxies clusters in the observable Universe through the analysis of the fluctuations of the Comptonization parameter, $y$, representing an important tool to probe clusters physics and evolution as well as cosmology ${ }^{18}$. In particular, the angular power spectrum of the $\mathrm{tSZ}^{19}, C_{\ell}^{\mathrm{tSZ}}=\int \mathrm{d} z[\mathrm{~d} V /(\mathrm{d} z \mathrm{~d} \Omega)] \int \mathrm{d} M(\mathrm{~d} n / \mathrm{d} M)\left|y_{\ell}(M, z)\right|^{2}$, depending on the halo mass function, $\mathrm{d} n / \mathrm{d} M$, and the squared amplitude of two dimensional Fourier transform of the electron pressure profile, $\left|y_{\ell}\right|^{2}$, integrated over the redshift, with the differential volume element, and over the masses, provides information on certain combinations of cosmological parameters ${ }^{20}$.

At large scales $\left(\ell \lesssim 10^{3}\right) C_{\ell}^{\mathrm{tSZ}}$ reflects the Poisson random distribution of the sources and the evolution of the halo mass function related to the amplitude of matter clustering, i.e., to $\sigma_{8}$. The electron pressure profile is instead relevant at all scales, affecting the $C_{\ell}^{\mathrm{tSZ}}$ amplitude and, at smaller scales, the $C_{\ell}^{\mathrm{tSZ}}$ shape, because of the halos morphology, resulting in a peak in $\ell(\ell+1) C_{\ell}^{\mathrm{tSZ}}$ at $\ell \simeq 3000$. The determination of the electron pressure profile dependence on the cluster mass is based on the relation between the SZ flux and the mass. The latter is typically inferred from X-ray observations and suffers from a relevant mismatch with that derived from lensing observations, when available, that are in principle sensitive to the true cluster mass, thus calling for the introduction of a bias parameter, $B=(1-b)^{-1}$, in the formula of the electron pressure profile, $(1-b)$ being the usual mass bias factor accounting for any difference between the X-ray mass proxies used to establish the scaling relations and the true (halo) mass.

At $\ell \lesssim 10^{3}, C_{\ell}^{\mathrm{tSZ}}$ is found to be mainly dependent on the parameter combination $F=\sigma_{8}\left(\Omega_{\mathrm{m}} / B\right)^{0.40} h^{-0.21}$ through the approximate relation $\ell(\ell+1) C_{\ell}^{\mathrm{tSZ}} \propto F^{8.1}$, and $F$ can be then estimated from data with likelihood methods including also proper parametrizations of noise and the other ("foreground") contributions to $C_{\ell}$. The value $F=0.460 \pm 0.012$ at $68 \%$ CL retrieved in this way can be compared with that of $F B^{0.40}=0.568 \pm 0.015$ at $68 \%$ CL derived from Planck CMB temperature and low- $\ell$ polarization anisotropy for the base $\Lambda$ CDM model in the second release ${ }^{3}$ (or only slightly changed when using the values in Tab. 1), thus requiring $B=$ $1.71 \pm 0.17$ at $68 \%$ CL, i.e. $1-b \simeq 0.58$, consistent with results from SZ number counts $^{21}$, i.e. the mass estimated from X-rays is about $40 \%$ lower than their true mass $^{22}$. Including deviations from hydrostatic equilibrium by non-thermal pressure could partially alleviate this problem, while forthcoming X-ray measurements by, e.g., eROSITA, will greatly reduce uncertainties in X-ray data and, together with next SZ observations, e.g., from NIKA2, could solve this bias problem, making galaxies clusters competitive to constrain some cosmological parameters, or, maybe, reveal new physics ${ }^{20}$. 


\section{Future CMB missions}

Recent proposals of CMB missions are based on concepts and designs aimed at improving the measure of polarization anisotropies and at minimizing the impact of foregrounds using a large number of frequency channels (see also the CM1 session, and related contributions in these proceedings, for their complementarities with ground-based and balloon-borne projects).

The Primordial Inflation Explorer ${ }^{23}$ (PIXIE) is an Explorer-class mission submitted to NASA in 2011 aimed at the mapping with high signal to noise ratio in absolute intensity and linear polarization the CMB and the diffuse astrophysical foregrounds over the full sky, from $30 \mathrm{GHz}$ to $6 \mathrm{THz}(1 \mathrm{~cm}$ to $50 \mu \mathrm{m})$ with degree resolution. It is designed to perform absolute spectroscopy measurements and to simultaneously observe the large-scale CMB B-modes. It will provide crucial constraints on Universe ionization history, on CIB spectrum and anisotropies. Great hopes on CMB spectral distortion parameters (see also the CM3 session and related contributions in these proceedings) are expected from PIXIE, thanks to its absolute calibration of the CMB temperature measure with a precision about $10^{3}$ times better than COBE/FIRAS.

LiteBIRD $^{24}$ is a degree resolution Lite (Light) Satellite for the studies of Bmode polarization and Inflation from cosmic background Radiation Detection at the extremely early Universe proposed to the Japan Aerospace Exploration Agency (JAXA) in 2015. It is a highly-targeted mission, with the primary aim of the Bmodes detection at the level of $r \sim 10^{-3}$, about one order of magnitude lower than that achievable from the ground, performed in combination with ground based, highresolution telescopes helping the accurate subtraction of the lensing contribution to B-mode.

The Cosmic ORigins Explorer (CORE) ${ }^{25}$, submitted to the ESA in 2016 in response to a call for future medium-sized space mission proposals for the M5 launch opportunity of ESA Cosmic Vision programme, is a satellite dedicated to microwave polarization. CORE is targeted to provide and exploit definitive maps of CMB polarization anisotropies at large and medium angular scales, with a suppression of all systematic effects at an extremely accurate level over 19 frequency channels spanning the $60-600 \mathrm{GHz}$ range, relevant to characterize astrophysical foreground emissions.

The Probe of Inflation and Cosmic Origin (PICO) ${ }^{26}$ is currently one of the 8 Probe-Scale - $\$ 400 \mathrm{M}-\$ 1000 \mathrm{M}$ - space missions proposed to NASA whose study is being funded. The concept and performance of PICO are similar to those of CORE regarding $\mathrm{CMB}$ polarization anisotropies, but considering also the option of adding CMB spectrum absolute measurements ${ }^{\mathrm{c}}$ with a sensitivity similar or slightly better than that proposed for PIXIE.

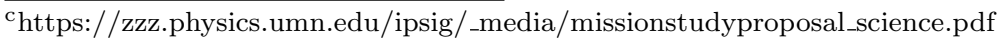


CMB Bharat ${ }^{\mathrm{d}}$ is a satellite, proposed in response to the Announcement of Opportunity for future Astronomy missions by the Indian Space Research Organisation (ISRO), dedicated to microwave polarization with concept and performance similar to those of CORE but with a proposed frequency coverage up to about $900 \mathrm{GHz}$ to further improve CIB science and foreground mitigation, and, possibly, equipped with a dedicated spectrometer to perform absolute spectrum measurements and useful also for high level calibration of the differential polarization signal.

Remarkably, the next generation of CMB missions, and, if funded, a large mission, as, e.g., in the view proposed for the Polarized Radiation Imaging and Spectroscopy Mission (PRISM) ${ }^{27}$, far from being limited to the scientific outcomes driven by their main goals, will allow us to significantly improve the current results in parameter estimation and large scale structure, to characterize a wide set of cosmological model extensions, to answer fundamental physics questions, and, in the case of a detection of primordial gravitational waves, to determine the shape of their spectrum with great implications for our understanding of the early Universe (see, e.g., refs. ${ }^{28,29}$ for detailed forecasts based on CORE specifications), offering at the same time exquisite all-sky surveys for astrophysical studies, both Galactic and extragalactic (see Sect. 5.1).

\section{Astrophysical cosmology}

\subsection{Thermal and star formation history from cosmic dipoles}

The analysis of cosmic dipoles is crucial in cosmology, being linked with the isotropy and homogeneity of the Universe at the largest scales. The observed dipole comes from different contributions, of both cosmological and astrophysical (extragalactic and Galactic) origin. The frequency spectral behaviour of the dipole effect due to the observer motion with respect to the CMB rest frame offers the opportunity to be investigated to constrain the $\mathrm{CMB}$ and $\mathrm{CIB}$ frequency spectra thanks to future missions, described in Sect. 3, without requiring an independent absolute calibration (see Trombetti, these proceedings).

\subsection{1. $C M B$ and CIB spectra: from monopoles to dipoles}

The precise interpretation of tiny CMB spectral distortions predicted at different cosmic times provides information on cosmological and astrophysical processes, some of them unavoidable, otherwise unexplored. The analysis of CIB spectrum, still not well known, can provide instead a better understanding of the dust-obscured star-formation phase of galaxy evolution. Since FIRAS, no remarkable improvements have been achieved in the knowledge of CMB spectrum at $\nu \gtrsim 30 \mathrm{GHz}$, while, in spite of its absolute calibration precision of $0.57 \mathrm{mK}$, the FIRAS characterization CIB amplitude and shape still presents a substantial uncertainty.

dhttp://cmb-bharat.in/ 
At early times, a Bose-Einstein (BE) like distorted spectrum with a positive (dimensionless) chemical potential, $\mu$, is produced by the dissipation of primordial perturbations at small scales ${ }^{30,31}$. Moreover, the matter temperature faster decrease with respect to that of radiation temperature in an expanding Universe produces a spectral distortion mainly characterized by a BE-like spectrum with a negative chemical potential. The chemical potential quantifies the fractional energy, $\Delta \varepsilon / \varepsilon_{i}$, exchanged in the plasma during the interaction; for small distortions, $\mu \simeq 1.4 \Delta \varepsilon / \varepsilon_{i}$. The photon occupation number of the BE spectrum is $\eta_{B E}=1 /\left(e^{x_{e}+\mu}-1\right)$, where $x_{e}=x / \phi(z), \phi(z)=T_{e}(z) / T_{C M B}(z)$, being $T_{e}(z)$ the electron temperature, $T_{C M B}=T_{0}(1+z)$ and $x=h \nu /\left(k T_{C M B}\right)$ a redshift independent, dimensionless frequency. The electron heating during the cosmological reionization associated with the early stages of structure and star formation is responsible for late type distortions, described, at high frequency, by a Comptonization spectrum ${ }^{32}$ with Comptonization parameter $u(t)=\int_{t_{i}}^{t}\left[\left(\phi-\phi_{i}\right) / \phi\right]\left(k_{B} T_{e} / m_{e} c^{2}\right) n_{e} \sigma_{T} c d t$. For small energy injections and integrating over the relevant epochs $u \simeq(1 / 4) \Delta \varepsilon / \varepsilon_{i}$. CMB spectrum studies can also constrain many types of non-standard processes, i.e. non evaporating $\mathrm{BH}$ spin, small scale magnetic fields power spectra, vacuum energy density and particle decay.

The analytic form of the CIB spectrum, observed at present time, is ${ }^{33} \eta_{C I B}=$ $\left[c^{2} /\left(2 h \nu^{3}\right)\right] I_{C I B}(\nu)=I_{0}\left[k_{B} T_{C I B} /\left(h \nu_{0}\right)\right]^{k_{F}} x_{C I B}^{k_{F}} /\left(e^{x_{C I B}}-1\right)$, with $T_{C I B}=(18.5 \pm$ 1.2) $\mathrm{K}, x_{C I B}=h \nu / k_{B} T_{C I B}=7.78\left(\nu / \nu_{0}\right), \nu_{0}=3 \times 10^{12} \mathrm{~Hz}$ and $k_{F}=0.64 \pm 0.12$. Here $I_{0}$ sets the CIB spectrum amplitude, its best-fit value being $(1.3 \pm 0.4) \times$ $10^{-5}$. Indeed, the direct determination of the CIB spectrum is not trivial, requiring absolute intensity measurements and being limited by foreground signals.

A relative velocity between an observer and the CMB rest frame induces a dipole (the so called $\ell=1$ anisotropy) in the CMB sky temperature through the Doppler effect, dominated by the velocity of the Solar System, $\overrightarrow{\beta_{S}}=\overrightarrow{v_{S}} / \vec{c}$, with respect to the CMB (Solar dipole), with a seasonal modulation due to the velocity of the Earth or the satellite, $\overrightarrow{\beta_{o}}$, with respect to the Sun (orbital dipole). Neglecting the orbital dipole (useful for calibration aims), we will denote with $\vec{\beta}$ the relative velocity of the Solar dipole.

The dipole amplitude is directly proportional to the first (logarithmic) derivative with respect to the frequency of the photon occupation number ${ }^{34}$. More in general, the full pattern at a given observational frequency, $\nu_{o b s}$, can be computed from the photon distribution function, $\eta^{B B \text {,dist }}$, for the assumed type of distortions (BB, CIB, $\mathrm{BE}$ or Comptonization $(\mathrm{C}))$ at the frequency $\nu_{\text {obs }}$ multiplied by $(1-\hat{n} \cdot \vec{\beta}) /\left(1-\beta^{2}\right)^{1 / 2}$ to account for all the possible sky directions with respect to the observer peculiar velocity $^{35}$. The observed signal map in thermodynamic temperature is given by $T_{\text {therm }}^{B B / \text { dist }}(\nu, \hat{n}, \vec{\beta})=x T_{0} / \ln \left(1 /\left(\eta(\nu, \hat{n}, \vec{\beta})^{B B, \text { dist }}+1\right)\right)$, where $\eta(\nu, \hat{n}, \vec{\beta})=\eta\left(\nu^{\prime}\right)$ with $\nu^{\prime}=\nu\left((1-\hat{n} \cdot \vec{\beta}) /\left(1-\beta^{2}\right)^{1 / 2}\right.$. Decomposing the maps into spherical harmonics and reproducing them from the $a_{\ell m}$ up to a desired multipole $\ell_{\max }$, it is possible to compute the signal map for each $\ell$ : at the accuracies of the discussed projects, it is 
found to be important for the dipole, possibly appreciable for the quadrupole and of strongly decreasing relevance at higher multipoles.

\subsubsection{Results and perspectives}

Detailed simulations allow us to quantify the sensitivity of a given project to measure spectral distortion parameters and CIB amplitude in the presence of only ideal noise or including also potential (calibration and foreground) residuals through $\Delta \chi^{2}$ analyses based on the comparison of the adopted input sky with different model assumptions, possibly applying suitable sky masks like those publicly available from the Planck Legacy Archive (PLA) ${ }^{\mathrm{e}}$. Calibration errors and foreground residuals can be modeled generating random fluctuations, depending also on sky signal, parametrizing their overall fractional levels at a certain pixel scale with two key parameters (namely $E_{\text {cal }}$ and $E_{\text {for }}$ ).

The joint exploitation of all frequency channels (assumed independent from each other) and of the combinations of the maps differences from pairs of frequency bands is found to be particularly advantageous, being sensitive to both amplitude and spectral shape of the considered dipole signal, while the use of a suitable mask can improve parameters reconstruction. Tab. 2 summarizes the main results, obtained for the CORE specifications, expressed in terms of a factor characterizing the improvement with respect to FIRAS. Even under pessimistic assumptions, a

\footnotetext{
ehttp://pla.esac.esa.int/pla/
}

Table 2. Predicted improvement in the recovery of CIB spectrum amplitude and the CMB distortion parameters with respect to FIRAS for different calibration and foreground residual assumptions. "P76" stands for the Planck common mask (in temperature) of the PR2-2015 release covering about $76 \%$ of the sky and "P76ext" denotes its extension that excludes all the pixels at $|b| \leq 30^{\circ}$. When not stated, all values refer to $E_{\text {cal }}$ and $E_{\text {for }}$ at $N_{\text {side }}=64$; the values in round brackets possibly refer to the corresponding frequency range (in $\mathrm{GHz}$ ). From ref. ${ }^{35}$ [CSISSA Medialab Srl. Reproduced by permission of IOP Publishing. All rights reserved].

\begin{tabular}{c|c|c|c|c|c}
\hline & $E_{\text {cal }}(\%)$ & $E_{\text {for }}(\%)$ & CIB amplitude & BE & C \\
\hline Ideal case, all sky & - & - & $\simeq 4.4 \times 10^{3}$ & $\simeq 10^{3}$ & $\simeq 6.0 \times 10^{2}$ \\
\hline All sky & $10^{-4}$ & $10^{-2}$ & $\simeq 15$ & $\simeq 42$ & $\simeq 18$ \\
P76 & $10^{-4}$ & $10^{-2}$ & $\simeq 19$ & $\simeq 42$ & $\simeq 18$ \\
P76ext & $10^{-2}$ & $10^{-2}$ & $\simeq 17$ & $\sim 4$ & $\sim 2$ \\
P76ext & $10^{-4}$ & $10^{-2}$ & $\simeq 22$ & $\simeq 47$ & $\simeq 21$ \\
P76ext & $10^{-4}$ & $10^{-3}$ & $\simeq 2.1 \times 10^{2}$ & $\simeq 2.4 \times 10^{2}$ & $\simeq 1.1 \times 10^{2}$ \\
P76ext & $10_{(\leq 295)}^{-10^{-2}(\geq 340)}$ & $10^{-2}$ & $\simeq 19$ & $\simeq 26$ & $\simeq 11$ \\
P76ext & $10_{(\leq 295)}^{-1}-10_{(\geq 340)}^{-2}$ & $10^{-3}$ & $\simeq 48$ & $\simeq 35$ & $\simeq 15$ \\
\hline P76ext, $N_{\mathrm{s}}=128$ & $10_{(\leq 295)}^{-1}-10_{(\geq 340)}^{-2}$ & $10^{-2}$ & $\simeq 38$ & $\simeq 51$ & $\simeq 23$ \\
P76ext, $N_{\mathrm{s}}=128$ & $10_{(\leq 295)}^{-1}-10_{(\geq 340)}^{-2}$ & $10^{-3}$ & $\simeq 43$ & $\simeq 87$ & $\simeq 39$ \\
P76ext, $N_{\mathrm{s}}=256$ & $10_{(\leq 295)}^{-1}-10_{(\geq 340)}^{-2}$ & $10^{-2}$ & $\simeq 76$ & $\simeq 98$ & $\simeq 44$ \\
P76ext, $N_{\mathrm{s}}=256$ & $10_{(\leq 295)}^{-3}-10_{(\geq 340)}^{-2}$ & $10^{-3}$ & $\simeq 85$ & $\simeq 1.6 \times 10^{2}$ & $\simeq 73$ \\
\hline
\end{tabular}


clear improvement in the CIB spectrum amplitude and a marginal detection of the energy release associated to astrophysical reionization models are foreseen, while improvements in foreground mitigation and calibration will allow us to derive more precise results or constraints on dissipation mechanisms occurring at any epoch, including primeval processes.

\subsection{1-cm signal of neutral hydrogen from high cosmological redshifts}

The evolution history of the neutral Universe in the first few hundred million years after the Big Bang, as well as the processes of the primordial star formation and reionization, remains poorly constrained. Theoretical modelling bridges between existing observations of the two dimensional surface of recombination at redshift $z \sim 1100$ probed by the CMB radiation, and the epoch of galaxy formation with the most distant detected galaxy located at $z=11.1^{36}$.

The 21-cm signal of neutral hydrogen provides a unique way to constrain the first few hundred million years (see Fialkov, these proceedings). The hyper-fine splitting of the lowest hydrogen energy level gives rise to the rest-frame $\nu_{21}=1.42$ $\mathrm{GHz}$ radio signal with the equivalent wavelength of $21 \mathrm{~cm}$ (see ref. ${ }^{37}$ for a recent review). The cumulative signal of neutral intergalactic medium observed against the background radiation depends on the processes of cosmic heating and ionization and, to the leading order, scales as $T_{21} \propto x_{\mathrm{HI}}\left(1-T_{\mathrm{rad}} T_{\mathrm{S}}^{-1}\right)$ where $T_{\text {rad }}$ is the brightness temperature of the background radiation at $1.42 \mathrm{GHz}$ and $T_{\mathrm{S}}$ is the spin temperature of the transition which at cosmic dawn is close to the kinetic temperature of the gas, $T_{\mathrm{K}}$. Owing to its dependence on the underlying astrophysics and cosmology, this signal is a powerful tool to characterize the formation and the evolution of the first populations of astrophysical sources and, potentially, properties of dark matter (DM), across cosmic time. Uncertainties in the high-redshift astrophysical processes result in a large variety of the possible $21-\mathrm{cm}$ signals (see grey lines in Fig. 1).

Observations with the High-Band antenna of the Experiment to Detect the Global EoR Signature (EDGES) ${ }^{39-41}$ and the Shaped Antenna measurement of the background RAdio Spectrum2 (SARAS2) ${ }^{42,43}$ yield upper limits on the 21-cm signal from redshifts $z \sim 6-15$. In the framework of standard astrophysical modelling, the data require star formation in small DM halos ${ }^{41}$ and rule out extremely non-efficient X-ray heating sources ${ }^{41-43}$.

Recently, the first detection of the sky-averaged (global) 21-cm signal of neutral hydrogen from $z \sim 13-27$ has been claimed based on two years of observations with the EDGES Low-Band antenna in the $50-100 \mathrm{MHz}$ frequency range ${ }^{44}$. If confirmed, this is the only existing observation from the intermediate redshift range and is the first observational evidence of the primordial star formation at $z \sim 20$ ( 180 million years after the Big Bang) and early X-ray heating. The reported cosmological 21-cm signal is centered at $z=17.2$ (which corresponds to $\nu=78.2$ $\mathrm{MHz}$ ), and features an absorption trough of $T_{21}=-500_{-500}^{+200} \mathrm{mK}$, where the error 


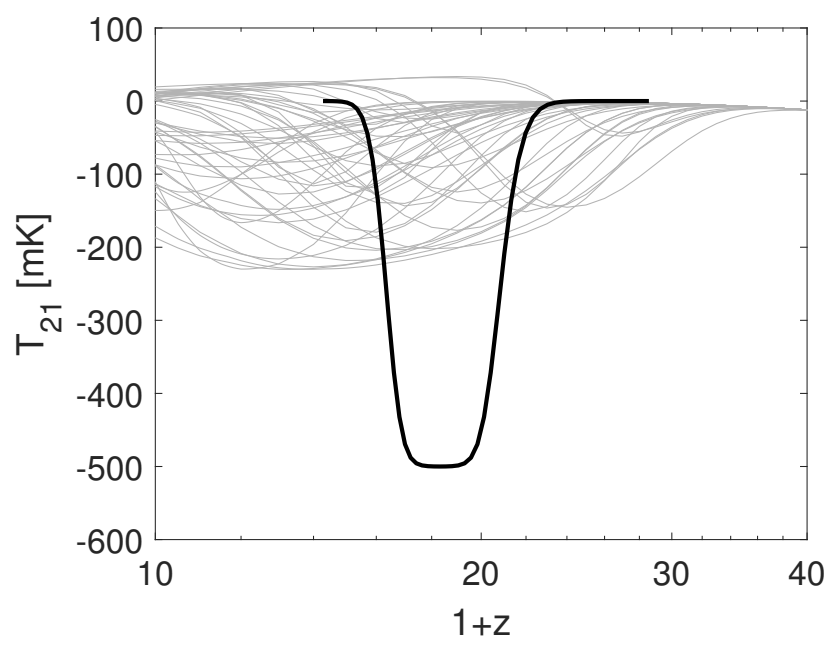

Fig. 1. The variety of plausible predicted 21-cm signals (grey lines - computed here for 50 models according to ref. ${ }^{38}$ ) and the best-fit profile of the EDGES Low-band detection (thick black line).

corresponds to $99 \%$ confidence including both thermal and systematic noise. The depth of the feature is at least twice as strong as predicted in standard astrophysical scenarios (based on the assumption of $\Lambda \mathrm{CDM}$ cosmology and hierarchical structure formation) where the strongest possible feature at $z \simeq 17$ is $\simeq-209 \mathrm{mK}$, assuming the $\mathrm{CMB}$ as the background radiation with $T_{C M B} \simeq 49.5 \mathrm{~K}$ and the coldest possible temperature of the intergalactic medium (IGM) of $\sim 7.2 \mathrm{~K}$ obtained in the absence of X-ray heating sources. The observed $T_{21}<-300 \mathrm{mK}$ requires either the gas to be much colder (around $5.2 \mathrm{~K}$ ) or the background radiation to be much stronger (around $67.2 \mathrm{~K}$ ), which is hard to explain by astrophysics alone. The frequency of the detected feature can be related to the formation of the first stars which couple the spin temperature to the temperature of the gas via absorption and re-emission of Ly- $\alpha$ photons; while the narrowness of the feature indicates a relatively rapid onset of X-ray heating.

To explain the depth of the reported feature exotic mechanisms have to be added to the standard picture. The amplitude of the observed $21-\mathrm{cm}$ feature can be explained by non-gravitational interaction between DM and baryons, e.g., via Rutherford-like scattering which could drain excess energy from the gas lowering its kinetic temperature (see, e.g., ref. ${ }^{45}$ ). Even though this scenario is strongly constrained by observations, it is not completely ruled out and is still plausible for a narrow range of parameters including the DM mass, electric charge of DM particle and cross-section (see, e.g., ref. ${ }^{46}$ ). A smoking gun signature of the baryon-dark matter (b-dm) scattering with a velocity-dependent cross-section, first proposed in ref. ${ }^{45}$ as a solution to the EDGES Low-Band anomaly, was shown to be an enhanced pattern of $\mathrm{BAO}$ in the $21-\mathrm{cm}$ power spectrum ${ }^{47}$. The power spectrum itself is 
boosted by as much as three orders of magnitude which renders the fluctuations detectable by telescopes such as the low band antennas of LOFAR.

An excess radio background at the rest-frame $1.42 \mathrm{GHz}$ at $z=17$ would also explain the large contrast observed between the background temperature and the spin temperature of the $21-\mathrm{cm}$ transition ${ }^{44,48}$. Interestingly, evidence of excess radio background above the CMB at low radio frequencies was detected by ARCADE2 at $3-90 \mathrm{GHz}^{49}$ and recently confirmed by LWA1 at $40-80 \mathrm{MHz}^{50}$. However, it is still not clear what part of the observed excess is extragalactic ${ }^{51}$. If it exists, the excess radio background is also expected to boost the $21-\mathrm{cm}_{\text {power }}$ spectrum $^{52}$.

Verification of the EDGES-Low signal is on the way with instruments such as the EDGES Mid-Band antenna, SARAS3, LEDA, and the LOFAR Low Band.

\subsubsection{Black holes high mass X-ray binaries microquasars and cosmic backgrounds}

A possible explanation of the EDGES excess involving black hole high mass X-ray binary microquasars (BH-HMXB-MQs) has been recently proposed in ref. ${ }^{53}$. There is indeed an increasing evidence of a high formation rate of BH-HMXBs at high redshifts, theoretically advanced in ref. ${ }^{54}$. According to this model, a significant fraction of Pop III stars with mass $\gtrsim 20 \mathrm{M}_{\odot}$ and low metallicity, $\mathrm{Z} \leq 0.001 \mathrm{Z} \odot$, in binaries or larger multiple systems is predicted to collapse with no energetic SN kicks and to remain in situ, finally resulting into BH-HMXB-MQs of Pop III. These objects are likely sources of a substantial synchrotron radio emission, with a relatively smooth spectrum, able to produce a cosmic background that could significantly enhance the absorption feature of the redshifted $21-\mathrm{cm}$ line by neutral hydrogen produced at redshifts $z \sim 17$. The absence of a cosmic far-IR thermal background associated with a cosmic radio background can be explained in this model by the fact that BH-HMXB-MQs in stellar clusters of Pop III are formed before the appearance of SN explosions, neutron stars and dust. BH-HMXB-MQs promptly inject hard X-rays and relativistic jets in the cold hydrogen that enshrouds the slowly expanding HII regions ionized by the most massive progenitor stars of Pop III. The high column depths for the cold hydrogen IGM largely block the Xrays from inner sources but are essentially transparent to the radio synchrotron emission.

Crucial tests of this scenario will be based on the next generation of multiwavelength / multi-messenger surveys, including interferometric observations of the HI redshifted 21-cm line able to provide an accurate tomographic view of HI inhomogeneities, X-ray missions that will allow us a better understanding of the HMXBs role, and gravitational waves observatories providing a larger statistics of HMXBs and BBHs useful for cosmological investigations and, hopefully, a measure of the spectrum of gravitational wave background that, in this model, is predicted to have a flattening of the spectral index at frequencies as low as $\sim 30 \mathrm{~Hz}$. 


\section{Extragalactic astrophysics}

\subsection{Extragalactic point sources}

Although the main goal of Planck was to provide accurate all-sky CMB temperature and polarisation maps, it has supplied unprecedented data of astrophysical interest in several fields. On the extragalactic side, Planck carried out the deepest systematic all-sky survey of galaxy clusters seen via the SZ effect. It also detected thousands of dusty galaxies as well as many hundreds of extragalactic radio sources in a spectral range difficult or impossible to explore from the ground and only lightly surveyed by other space missions.

Because of its quite limited angular resolution $\left(\simeq 5^{\prime}\right.$ at sub-mm wavelengths) Planck was confusion limited at quite bright flux density levels, so that the overwhelming majority of dusty galaxies it detected are very local, at distances generally below $100 \mathrm{Mpc}^{55}$. Planck offered the first opportunity to accurately determine their sub-mm properties ${ }^{56}$.

Even more interestingly, Planck opened new paths to the study of early phases of galaxy evolution, exploiting the strong gravitational lensing (Sect. 5.1.1), and of cluster evolution (Sect. 5.1.2); see De Zotti et al., these proceedings.

\subsubsection{Strongly lensed galaxies}

Somewhat unexpectedly (but see the predictions by ref. ${ }^{57}$ ) Planck also succeeded at detecting some high- $z$ galaxies whose flux densities were boosted by extreme gravitational magnifications ${ }^{58}$ (see the left panel of Fig. 2.)

The 11 strongly lensed galaxies discovered on Planck maps ${ }^{58}$ have redshifts in the range 2.2-3.6. This offers the exciting possibility of exploiting high spatial and spectral resolution follow-up to address major, still open issues on galaxy formation and evolution: which are the main physical mechanisms shaping the galaxy properties: in situ processes? interactions? mergers? cold flows from the intergalactic medium? How do feedback processes work? To settle these issues we need direct information on the structure and the dynamics of high- $z$ galaxies. But these are compact, with typical sizes of $1-2 \mathrm{kpc}$ (e.g., ref. ${ }^{59}$ ), corresponding to angular sizes of $0.1-0.2$ arcsec at $z \simeq 2-3$. Thus they are hardly resolved even by ALMA and by the HST. If they are resolved, high enough $\mathrm{S} / \mathrm{N}$ ratios per resolution element are achieved only for the brightest galaxies, probably not representative of the general population. Strong lensing offers a way out of all these difficulties.

\subsubsection{Proto-clusters}

Classical techniques for detecting galaxy clusters (optical/near-IR "red sequence", X-ray emission, SZ effect) preferentially or exclusively select evolved objects, with mature galaxy populations and a hot intra-cluster medium. As a result most known clusters are at $z<1.5$, i.e. below the peak of global star-formation activity. 

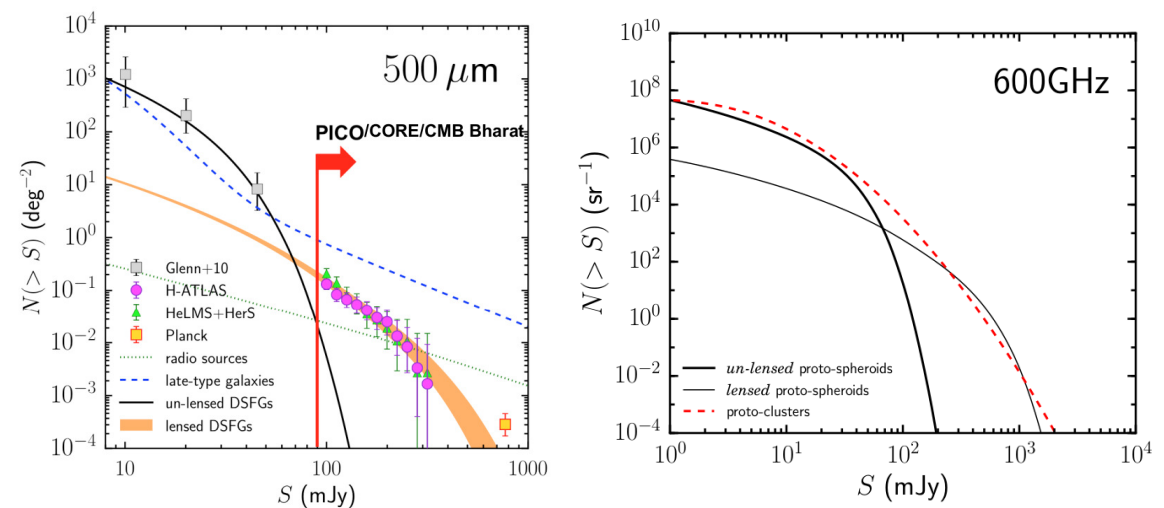

Fig. 2. Left panel: integral counts of the main extragalactic source populations at $500 \mu \mathrm{m}$ $(600 \mathrm{GHz})$. The counts of unlensed (solid black line) and strongly lensed (orange band) high- $z$ galaxies (labelled DSFGs) are compared with observational determinations from Herschel surveys. The yellow square on the bottom-right corner shows the estimated counts of strongly lensed galaxies detected by Planck. The dashed blue line shows the counts of late-type (normal plus starburst) galaxies. All these lines show predictions of the model by ref. ${ }^{60}$. The dotted green line shows the counts of radio sources predicted by the C2Ex model of ref. ${ }^{61}$. The vertical red line shows the estimated detection limits of planned next generation CMB experiments, PICO, CORE and CMB Bharat. Adapted from ref. ${ }^{62}$ [C)SISSA Medialab Srl. Reproduced by permission of IOP Publishing. All rights reserved]. Right panel: integral counts of proto-clusters at $600 \mathrm{GHz}$, predicted by the model of ref. ${ }^{63}$, compared with the counts of high- $z$ lensed and unlensed proto-spheroidal galaxies.

At higher redshifts cluster members enter the active star-formation phase and the hot intergalactic gas is no longer necessarily in place. But then strong galaxy overdensities stand out as intensity peaks in the low angular resolution maps of CMB experiments, as clearly demonstrated by Planck ${ }^{64}$. Thus CMB experiments offer the possibility of extending the investigation of cluster evolution up to much higher redshifts than would be possible by other means.

However, Planck's resolution was too poor to detect individual proto-clusters ${ }^{63}$. As illustrated by the right panel of Fig. 2, next generation experiments will detect many tens of thousands of these objects as peaks in sub-mm maps, in addition to the evolved ones, detected by the SZ effect. This will constitute a real breakthrough in the observational validation of models for structure formation.

\subsection{Virial clouds in the galactic halos}

The rotation of the galactic objects and disks of various nearby edge-on spiral galaxies has been studied by using the Doppler asymmetry in the CMB radiation ${ }^{65-70}$. It was proposed that the asymmetry is caused by molecular hydrogen clouds which are exactly merged with the CMB, i.e. they are at the CMB temperature ${ }^{71}$. An objection was raised that the effect might not be due to pure molecular hydrogen clouds, it might be due to clouds completely made of interstellar dust or clouds 
containing some fraction of $H_{2}$ and dust. In order to address the issue in this paper we only modeled the clouds with the assumption that they are composed of pure $\mathrm{H}_{2}$ molecules (single fluid model). After modeling the clouds we tried to estimate the luminosity caused by the clouds (see Qadir et al., these proceedings). Further, we want to extend the analysis (in a later work) to a two-fluid model. The hope is that by comparing it with the Planck data we can constrain the missing baryonic distribution in the galactic halos.

Since, the clouds are immersed in a heat bath which is CMB, so, they are isothermal and we called them "virial clouds". They are formed due to the Jeans instability, so, they should have a precise Jeans mass and radius. The density of the clouds should be flat at the center and then it goes exactly zero at the the Jeans radius in order to give a definite boundary. The density distribution can be given by the isothermal Lane-Emden equation but the density profile in that case will not be exactly zero at the boundary. In this case the density distribution had to be put on ad-hoc basis. We do not have to do this. Instead of using the isothermal Lane-Emden equation we used the canonical ensemble distribution to obtain the mass density profile. We also introduced the speed of sound $c_{s}$ which is given by $c_{s}=\sqrt{\left(\gamma k T_{C M B}\right) / m_{H}}$, where, $\gamma$ is the adiabatic factor. At the CMB temperature no higher degrees of freedom would excite, so, we only have the translational degree, making the ideal gas approximation extremely good and so $\gamma=5 / 3$. For our purpose, $m_{H} \approx 2.016 \mathrm{~g} / \mathrm{mol}$ and $T \approx 2.726 \mathrm{~K}$, so $c_{s} \simeq 1.110 \times 10^{4} \mathrm{~km} \mathrm{~s}^{-1}$. The virial theorem $2 K+U=0$ gives the Jeans mass squared as ${ }^{72}$

$$
M_{J}^{2} \simeq\left(\frac{81}{32 \pi \rho_{c}}\right)\left(\frac{3 c_{s}^{2}}{5 G}\right)^{3},
$$

and the corresponding Jeans radius squared as

$$
R_{J}^{2}=\frac{27 c_{s}^{2}}{20 \pi \rho_{c} G}
$$

where $\rho_{c}$ is the central density of the virial cloud. We obtained a differential equation as

$$
r \frac{d \rho(r)}{d r}-r^{2}\left(\frac{4 \pi G m_{H}}{k T_{C M B}}\right) \rho^{2}(r)-\rho(r) \ln \left(\frac{\rho(r)}{\zeta}\right)=0 .
$$

where, $\zeta=8 m_{H}^{5 / 2}\left(G \rho_{c} / 3 k T_{C M B}\right)^{3 / 2}$. We have the initial condition $\left.\rho^{\prime}(r)\right|_{r=0}=0$ and the boundary condition that $\rho\left(R_{J}\right)=0$. As such, we can solve it numerically. The resulting density profile is shown in Fig. 3. The obtained central density of the cloud is $\rho_{c} \simeq 1.60 \times 10^{-18} \mathrm{~kg} \mathrm{~m}^{-3}$, the radius is $R_{J} \simeq 0.030 \mathrm{pc}$, and the mass is $M_{J} \simeq 0.798 M_{\odot}$.

Since the virial clouds are at the CMB temperature, their luminosity is the CMB luminosity, except for their Doppler shift. Thus, we only need to look at the differential frequency shift, $\Delta \nu / \nu=-\left(v_{r o t} / c\right) \cos \theta$, where $v_{\text {rot }}$ is the galactic halo rotational velocity and the luminosity of a single cloud, $L_{c}$, could be given by 


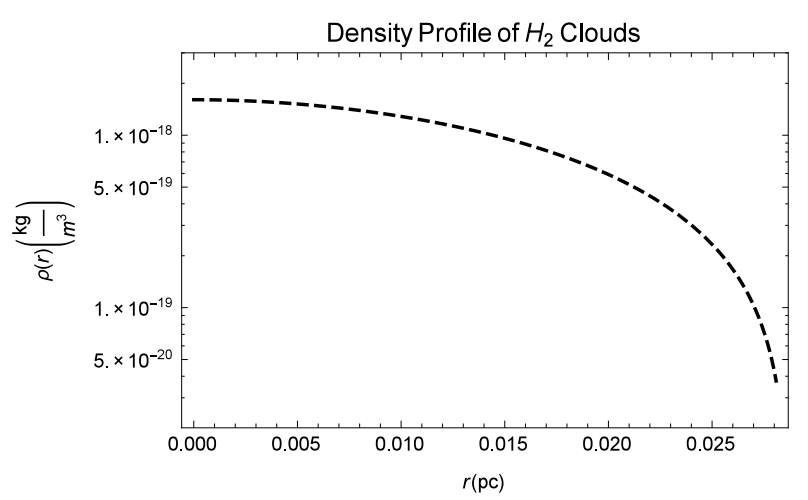

Fig. 3. The density of the virial molecular hydrogen cloud. It decreases monotonically from the central density, $\rho_{c} \simeq 1.60 \times 10^{-18} \mathrm{~kg} \mathrm{~m}^{-3}$, to zero at $r=R_{J} \simeq 0.030 \mathrm{pc}$.

$L_{c}=(\Delta \nu / \nu) L \simeq 1.486 \times 10^{-4} L \cos \theta$, being $\theta$ the angle between the direction of the velocity and the line of sight and $L$ the observed luminosity. This would directly give an estimate of the total number of clouds being seen.

\subsection{Perspectives from SPICA}

IR observations allowed us to study the obscured Universe, providing information about the physical state and energy balance of the cool matter relevant for the study of many physical processes occurring during the formation and evolution of galaxies and stars. Indeed, a large fraction ( $\sim 50 \%$ or more) of the energy emitted by stars in galaxies in form of optical and ultraviolet radiation is absorbed and reprocessed by dust grains and re-emitted in the IR, and, for distant objects, the cosmic expansion implies a further substantial redshift of the observed photons. For these reasons, galactic evolution, with a peak of activity at $\mathrm{z} \sim 1-4$ corresponding to a Universe age of about 2-3 Gyr, can be profitably investigated in the (mid- and far-) IR domain. Furthermore, improving our understanding of fundamental processes of star formation and evolution, including dust and gas physics of pre-stellar cores and proto-planetary discs, that can be more accurately studied in our galaxy in the IR band, allows us a better modeling of high- $z$ phenomena.

The physical conditions, such as excitation, density and metallicity, of nuclei of galaxies as well as of stars and planets formation regions can be investigated analyzing ionic, atomic, molecular and dust features in the IR. The star formation rate, the stellar type and the density of the gas in HII regions around hot young stars can be investigated using ionic fine structure lines, e.g., [NeII], [SIII], [OIII], while the AGN accretion rate is traced by spectral lines of highly ionized species, e.g., $[\mathrm{OIV}],[\mathrm{NeV}]$ (see the expected potentials of far-IR spectroscopic observations for studying galaxy/AGN co-evolution presented in ref. ${ }^{73}$ ). Photo-dissociation regions can be studied via the $[\mathrm{CII}]$ and $[\mathrm{OI}]$ lines and the emission from small dust grains 
and PAHs. Also, the cooling of diffuse warm gas in galaxies, through, e.g., [NII], probing ISM physics, is accessible to IR observations. Pure rotational lines from $\mathrm{H}_{2}$, $\mathrm{HD}$ and $\mathrm{OH}$, mid- to high-J $\mathrm{CO}$ and $\mathrm{H}_{2} \mathrm{O}$ lines, and PAH emission features appear in the object rest-frame IR band, the latter, containing several per cent of of the star-forming galaxies IR emission, being crucial for dust-obscured objects redshift determination. Other interesting dust features from minerals, e.g., olivine, calcite and dolomite, and $\mathrm{CO}_{2}$ ice, $\mathrm{C}_{2} \mathrm{H}_{2}$ molecules and fullerenes are also observable in the IR.

The above tracers have been only partially studied with previous IR missions, mainly because of limitations in telescope sizes and cryogenic performances.

To overcome these limits, a mid- and far-IR mission concept, the Space Infrared Telescope for Cosmology and Astrophysics (SPICA) ${ }^{74}$, was proposed to JAXA and ESA as a collaboration mission between Japanese and European scientists. It was selected in May 2018 by ESA as a finalist for the next Medium class Mission 5 of the Cosmic Vision programme for a proposed launch date of 2032. The spacecraft is designed to be deployed in a halo orbit around the Sun-Earth L2 Lagrange point to minimize systematic effects. The mission foresees a 2.5-meter diameter telescope cooled to below $8 \mathrm{~K}$. A combination of passive cooling and mechanical coolers, that will cool the telescope and the instruments, will enable a mission lifetime significantly beyond a nominal requirement of three years. Combining a large and cold telescope with instruments employing state-of-the-art ultra-sensitive detectors, an improvement in IR spectroscopic sensitivity (achieving the $-5 \sigma / 1 \mathrm{hr}-$ level of $\sim 5 \times 10^{-20} \mathrm{~W} / \mathrm{m}^{2}$ ) of more than two orders of magnitude with respect to Herschel, Spitzer and SOFIA is planned. SPICA will offer spectral resolving power ranging from $R \sim 50$ through 11000 in the 17-230 $\mu$ m domain as well as $R \sim 28.000$ spectroscopy between 12 and $18 \mu \mathrm{m}$. Furthermore, SPICA will perform efficient 30$37 \mu \mathrm{m}$ broad band mapping, and small field spectroscopic and polarimetric imaging in the 100-350 $\mu \mathrm{m}$ range.

A wide set of questions in astrophysics, e.g., the role in galaxy evolution of star formation, BHs and SNe explosions, metal and dust history, the matter cycle from galactic scale to larger structures and IGM, the formation of first galaxies and $\mathrm{BHs}$, will get benefit from deep analyses of the IR tracers made possible by such a mission.

\section{Acknowledgments}

CB, GDZ and TT acknowledge partial support from ASI/INAF agreement n. 2014024-R.1 for the Planck LFI Activity of Phase E2 and from the ASI/Physics Department of the university of Roma-Tor Vergata agreement n. 2016-24-H.0 for study activities of the Italian cosmology community; $\mathrm{MB}, \mathrm{CB}$ and TT acknowledge partial support from the INAF PRIN SKA/CTA project FORECaST; AF acknowledges the support by the Royal Society for her University Research Fellow at Sussex University; AQ and NT acknowledge the Salento University of Lecce for hospitality; AQ is grateful to IUPAP, ESA, ICRANet and AS-ICTP for support to participate 
in MG15. Some of the results in this paper have been derived using the HEALPix ${ }^{75}$ package. The use of the Planck Legacy Archive is acknowledged. It is a pleasure to thank all the speakers of the CM2 session and their collaborators.

\section{References}

1. J. A. Tauber, H. U. Norgaard-Nielsen, P. A. R. Ade et al., Planck pre-launch status: The optical system, $A \mathscr{G} A \mathbf{5 2 0}$, p. A2 (September 2010).

2. Planck Collaboration, Planck early results. I. The Planck mission, $A \& A$ 536, p. A1 (December 2011).

3. Planck Collaboration, Planck 2015 results. XIII. Cosmological parameters, A\&A 594, p. A13 (September 2016).

4. Planck Collaboration, Planck 2018 results. VI. Cosmological parameters, arXiv e-prints (July 2018).

5. Planck Collaboration, Planck 2018 results. I. Overview and the cosmological legacy of Planck, arXiv e-prints (July 2018).

6. Planck Collaboration, Planck 2018 results. II. Low Frequency Instrument data processing, arXiv e-prints (July 2018).

7. Planck Collaboration, Planck 2018 results. III. High Frequency Instrument data processing and frequency maps, arXiv e-prints (July 2018).

8. Planck Collaboration, Planck 2018 results. VIII. Gravitational lensing, arXiv e-prints (July 2018).

9. Planck Collaboration, Planck 2018 results. X. Constraints on inflation, arXiv e-prints (July 2018).

10. A. G. Riess, S. Casertano, W. Yuan, L. Macri, B. Bucciarelli, M. G. Lattanzi, J. W. MacKenty, J. B. Bowers, W. Zheng, A. V. Filippenko, C. Huang and R. I. Anderson, Milky Way Cepheid Standards for Measuring Cosmic Distances and Application to Gaia DR2: Implications for the Hubble Constant, ApJ 861, p. 126 (July 2018).

11. A. G. Riess, S. Casertano, W. Yuan, L. M. Macri and D. Scolnic, Large Magellanic Cloud Cepheid Standards Provide a 1\% Foundation for the Determination of the Hubble Constant and Stronger Evidence for Physics Beyond LambdaCDM, arXiv e-prints (March 2019).

12. A. E. Romano, Hubble trouble or Hubble bubble?, International Journal of Modern Physics D 27, p. 1850102 (2018).

13. G. P. da Silva and A. G. Cavalcanti, A More Accurate and Competitive Estimative of $\mathrm{H}_{0}$ in Intermediate Redshifts, Brazilian Journal of Physics 48, 521 (October 2018).

14. J. A. S. Lima and J. V. Cunha, A $3 \%$ Determination of $\mathrm{H}_{0}$ at Intermediate Redshifts, ApJL 781, p. L38 (February 2014).

15. C. D. Kreisch, F.-Y. Cyr-Racine and O. Doré, The Neutrino Puzzle: Anomalies, Interactions, and Cosmological Tensions, arXiv e-prints (February 2019).

16. V. Poulin, T. L. Smith, T. Karwal and M. Kamionkowski, Early Dark Energy 
Can Resolve The Hubble Tension, arXiv e-prints (November 2018).

17. Planck Collaboration, Planck 2015 results. XXVII. The second Planck catalogue of Sunyaev-Zeldovich sources, A\&A 594, p. A27 (September 2016).

18. Planck Collaboration, Planck 2015 results. XXII. A map of the thermal Sunyaev-Zeldovich effect, A\&A 594, p. A22 (September 2016).

19. E. Komatsu and T. Kitayama, Sunyaev-Zeldovich Fluctuations from Spatial Correlations between Clusters of Galaxies, ApJL 526, L1 (November 1999).

20. B. Bolliet, Cosmological constraints from the thermal Sunyaev Zeldovich power spectrum?, arXiv e-prints (June 2018).

21. Planck Collaboration, Planck 2015 results. XXIV. Cosmology from SunyaevZeldovich cluster counts, A\&A 594, p. A24 (September 2016).

22. B. Bolliet, B. Comis, E. Komatsu and J. F. Macías-Pérez, Dark energy constraints from the thermal Sunyaev-Zeldovich power spectrum, MNRAS 477, 4957 (July 2018).

23. A. Kogut, D. J. Fixsen, D. T. Chuss et al., The Primordial Inflation Explorer (PIXIE): a nulling polarimeter for cosmic microwave background observations, JCAP 7, p. 025 (July 2011).

24. H. Ishino, Y. Akiba, K. Arnold et al., LiteBIRD: lite satellite for the study of B-mode polarization and inflation from cosmic microwave background radiation detection, in Society of Photo-Optical Instrumentation Engineers (SPIE) Conference Series, , Proceedings of the SPIEVol. 9904July 2016.

25. J. Delabrouille, P. de Bernardis, F. R. Bouchet et al., Exploring cosmic origins with CORE: Survey requirements and mission design, JCAP 4, p. 014 (April 2018).

26. S. Hanany, M. Alvarez, E. Artis et al., PICO: Probe of Inflation and Cosmic Origins, arXiv e-prints (February 2019).

27. P. André, C. Baccigalupi, A. Banday et al., PRISM (Polarized Radiation Imaging and Spectroscopy Mission): an extended white paper, JCAP 2, p. 006 (February 2014).

28. E. Di Valentino, T. Brinckmann, M. Gerbino et al., Exploring cosmic origins with CORE: Cosmological parameters, JCAP 4, p. 017 (April 2018).

29. F. Finelli, M. Bucher, A. Achúcarro et al., Exploring cosmic origins with CORE: Inflation, JCAP 4, p. 016 (April 2018).

30. W. Hu, D. Scott and J. Silk, Power spectrum constraints from spectral distortions in the cosmic microwave background, ApJL 430, L5 (July 1994).

31. J. Chluba and R. A. Sunyaev, The evolution of CMB spectral distortions in the early Universe, MNRAS 419, 1294 (January 2012).

32. Y. B. Zel'dovich, A. F. Illarionov and R. A. Sunyaev, The Effect of Energy Release on the Emission Spectrum in a Hot Universe, Soviet Journal of Experimental and Theoretical Physics 35, p. 643 (1972).

33. D. J. Fixsen, E. Dwek, J. C. Mather, C. L. Bennett and R. A. Shafer, The Spectrum of the Extragalactic Far-Infrared Background from the COBE FIRAS 
Observations, ApJ 508, 123 (November 1998).

34. L. Danese and G. de Zotti, Dipole anisotropy and distortions of the spectrum of the cosmic microwave background, A\&A 94, p. L33 (February 1981).

35. C. Burigana, C. S. Carvalho, T. Trombetti et al., Exploring cosmic origins with CORE: Effects of observer peculiar motion, JCAP 4, p. 021 (April 2018).

36. P. A. Oesch, G. Brammer, P. G. van Dokkum et al., A Remarkably Luminous Galaxy at $\mathrm{z}=11.1$ Measured with Hubble Space Telescope Grism Spectroscopy, ApJ 819, p. 129 (March 2016).

37. R. Barkana, The rise of the first stars: Supersonic streaming, radiative feedback, and 21-cm cosmology, Physics Reports 645, 1 (July 2016).

38. A. Cohen, A. Fialkov, R. Barkana and M. Lotem, Charting the parameter space of the global 21-cm signal, MNRAS 472, 1915 (December 2017).

39. R. A. Monsalve, A. E. E. Rogers, J. D. Bowman and T. J. Mozdzen, Results from EDGES High-band. I. Constraints on Phenomenological Models for the Global 21 cm Signal, ApJ 847, p. 64 (September 2017).

40. R. A. Monsalve, B. Greig, J. D. Bowman, A. Mesinger, A. E. E. Rogers, T. J. Mozdzen, N. S. Kern and N. Mahesh, Results from EDGES High-band. II. Constraints on Parameters of Early Galaxies, ApJ 863, p. 11 (August 2018).

41. R. A. Monsalve, A. Fialkov, J. D. Bowman, A. E. E. Rogers, T. J. Mozdzen, A. Cohen, R. Barkana and N. Mahesh, Results from EDGES High-Band: III. New Constraints on Parameters of the Early Universe, arXiv e-prints (January 2019).

42. S. Singh, R. Subrahmanyan, N. Udaya Shankar, M. Sathyanarayana Rao, A. Fialkov, A. Cohen, R. Barkana, B. S. Girish, A. Raghunathan, R. Somashekar and K. S. Srivani, First Results on the Epoch of Reionization from First Light with SARAS 2, ApJL 845, p. L12 (August 2017).

43. S. Singh, R. Subrahmanyan, N. Udaya Shankar, M. Sathyanarayana Rao, A. Fialkov, A. Cohen, R. Barkana, B. S. Girish, A. Raghunathan, R. Somashekar and K. S. Srivani, SARAS 2 Constraints on Global $21 \mathrm{~cm}$ Signals from the Epoch of Reionization, ApJ 858, p. 54 (May 2018).

44. J. D. Bowman, A. E. E. Rogers, R. A. Monsalve, T. J. Mozdzen and N. Mahesh, An absorption profile centred at 78 megahertz in the sky-averaged spectrum, Nature 555, 67 (March 2018).

45. R. Barkana, Possible interaction between baryons and dark-matter particles revealed by the first stars, Nature 555, 71 (March 2018).

46. E. D. Kovetz, V. Poulin, V. Gluscevic, K. K. Boddy, R. Barkana and M. Kamionkowski, Tighter limits on dark matter explanations of the anomalous EDGES $21 \mathrm{~cm}$ signal, Phys.Rev.D 98, p. 103529 (November 2018).

47. A. Fialkov, R. Barkana and A. Cohen, Constraining Baryon-Dark-Matter Scattering with the Cosmic Dawn 21-cm Signal, Physical Review Letters 121, p. 011101 (July 2018).

48. C. Feng and G. Holder, Enhanced Global Signal of Neutral Hydrogen Due to 
Excess Radiation at Cosmic Dawn, ApJL 858, p. L17 (May 2018).

49. D. J. Fixsen, A. Kogut, S. Levin, M. Limon, P. Lubin, P. Mirel, M. Seiffert, J. Singal, E. Wollack, T. Villela and C. A. Wuensche, ARCADE 2 Measurement of the Absolute Sky Brightness at 3-90 GHz, ApJ 734, p. 5 (June 2011).

50. J. Dowell and G. B. Taylor, The Radio Background below $100 \mathrm{MHz}, A p J L \mathbf{8 5 8}$, p. L9 (May 2018).

51. R. Subrahmanyan and R. Cowsik, Is there an Unaccounted for Excess in the Extragalactic Cosmic Radio Background?, ApJ 776, p. 42 (October 2013).

52. A. Fialkov and R. Barkana, Signature of Excess Radio Background in the 21-cm Global Signal and Power Spectrum, arXiv e-prints (February 2019).

53. I. F. Mirabel, Black Hole High Mass X-ray Binary Microquasars at Cosmic Dawn, arXiv e-prints (February 2019).

54. I. F. Mirabel, M. Dijkstra, P. Laurent, A. Loeb and J. R. Pritchard, Stellar black holes at the dawn of the universe, $A \mathscr{E} A$ 528, p. A149 (April 2011).

55. M. Negrello, M. Clemens, J. Gonzalez-Nuevo et al., The local luminosity function of star-forming galaxies derived from the Planck Early Release Compact Source Catalogue, MNRAS 429, 1309 (February 2013).

56. M. S. Clemens, M. Negrello, G. De Zotti et al., Dust and star formation properties of a complete sample of local galaxies drawn from the Planck Early Release Compact Source Catalogue, MNRAS 433, 695 (July 2013).

57. M. Negrello, F. Perrotta, J. González-Nuevo, L. Silva, G. de Zotti, G. L. Granato, C. Baccigalupi and L. Danese, Astrophysical and cosmological information from large-scale submillimetre surveys of extragalactic sources, MNRAS 377, 1557 (June 2007).

58. R. Cañameras, N. P. H. Nesvadba, D. Guery et al., Planck's dusty GEMS: The brightest gravitationally lensed galaxies discovered with the Planck all-sky survey, $A \& A$ 581, p. A105 (September 2015).

59. S. Fujimoto, M. Ouchi, K. Kohno et al., ALMA 26 Arcmin $^{2}$ Survey of GOODS$\mathrm{S}$ at One Millimeter (ASAGAO): Average Morphology of High-z Dusty Starforming Galaxies in an Exponential Disk ( $n \simeq 1$ ), ApJ 861, p. 7 (July 2018).

60. Z.-Y. Cai, A. Lapi, J.-Q. Xia, G. De Zotti, M. Negrello, C. Gruppioni, E. Rigby, G. Castex, J. Delabrouille and L. Danese, A Hybrid Model for the Evolution of Galaxies and Active Galactic Nuclei in the Infrared, $A p J$ 768, p. 21 (May 2013).

61. M. Tucci, L. Toffolatti, G. de Zotti and E. Martínez-González, High-frequency predictions for number counts and spectral properties of extragalactic radio sources. New evidence of a break at mm wavelengths in spectra of bright blazar sources, A\&A 533, p. A57 (September 2011).

62. G. De Zotti, J. González-Nuevo, M. Lopez-Caniego et al., Exploring cosmic origins with CORE: Extragalactic sources in cosmic microwave background maps, JCAP 4, p. 020 (April 2018).

63. M. Negrello, J. Gonzalez-Nuevo, G. De Zotti, M. Bonato, Z.-Y. Cai, 
D. Clements, L. Danese, H. Dole, J. Greenslade, A. Lapi and L. Montier, On the statistics of proto-cluster candidates detected in the Planck all-sky survey, MNRAS 470, 2253 (September 2017).

64. Planck Collaboration Int. XXXIX, Planck intermediate results. XXXIX. The Planck list of high-redshift source candidates, $A \mathscr{E} A$ 596, p. A100 (December 2016).

65. F. de Paolis, V. G. Gurzadyan, G. Ingrosso, P. Jetzer, A. A. Nucita, A. Qadir, D. Vetrugno, A. L. Kashin, H. G. Khachatryan and S. Mirzoyan, Possible detection of the M 31 rotation in WMAP data, $A \& \& A$ 534, p. L8 (October 2011).

66. F. De Paolis, V. G. Gurzadyan, A. A. Nucita, G. Ingrosso, A. L. Kashin, H. G. Khachatryan, S. Mirzoyan, E. Poghosian, P. Jetzer, A. Qadir and D. Vetrugno, Planck confirmation of the disk and halo rotation of M 31, A\&A 5 565, p. L3 (May 2014).

67. F. De Paolis, V. G. Gurzadyan, A. A. Nucita, G. Ingrosso, A. L. Kashin, H. G. Khachatryan, S. Mirzoyan, G. Yegorian, P. Jetzer, A. Qadir and D. Vetrugno, Planck revealed bulk motion of Centaurus A lobes, $A \& \& A$ 580, p. L8 (August 2015).

68. V. G. Gurzadyan, F. De Paolis, A. A. Nucita, G. Ingrosso, A. L. Kashin, H. G. Khachatryan, S. Sargsyan, G. Yegorian, P. Jetzer, A. Qadir and D. Vetrugno, Planck view of the M 82 galaxy, $A \& A$ A 582, p. A77 (October 2015).

69. F. De Paolis, V. G. Gurzadyan, A. A. Nucita, L. Chemin, A. Qadir, A. L. Kashin, H. G. Khachatryan, S. Sargsyan, G. Yegorian, G. Ingrosso, P. Jetzer and D. Vetrugno, Triangulum galaxy viewed by Planck, $A \& A$ 593, p. A57 (September 2016).

70. V. G. Gurzadyan, F. De Paolis, A. A. Nucita, A. L. Kashin, A. Amekhyan, S. Sargsyan, G. Yegorian, A. Qadir, G. Ingrosso, P. Jetzer and D. Vetrugno, Messier 81's Planck view versus its halo mapping, A\&A 609, p. A131 (February 2018).

71. F. de Paolis, G. Ingrosso, P. Jetzer, A. Qadir and M. Roncadelli, Observing molecular hydrogen clouds and dark massive objects in galactic halos., $A \& A$ 299, p. 647 (July 1995).

72. S. Chandrasekhar, An introduction to the study of stellar structure. 1957.

73. M. Bonato, M. Negrello, Z.-Y. Cai et al., Exploring the relationship between black hole accretion and star formation with blind mid-/far-infrared spectroscopic surveys, MNRAS 444, 3446 (November 2014).

74. P. R. Roelfsema, H. Shibai, L. Armus et al., SPICA-A Large Cryogenic Infrared Space Telescope: Unveiling the Obscured Universe, PASA 35, p. e030 (August 2018).

75. K. M. Górski, E. Hivon, A. J. Banday, B. D. Wandelt, F. K. Hansen, M. Reinecke and M. Bartelmann, HEALPix: A Framework for High-Resolution Discretization and Fast Analysis of Data Distributed on the Sphere, ApJ 622, 759 (April 2005). 